\title{
Respuesta Glucémica e Insulínica de Pacientes con Diabetes Tipo 2 al consumo de Sopa de Calabaza Criolla (Cucúrbita Pepo L.) Enriquecida con Almidón de Banano
}

\author{
Angel E. Torres-Zapata ${ }^{(1)}$, María A. Aparicio-Trápala ${ }^{(1) *}$, Jorge L. Blé- Castillo $^{(2)}$, \\ Carlos A. Corzo-Sosa ${ }^{(1)}$ \\ Universidad Juárez Autónoma de Tabasco, (1) División Académica de Ciencias Agropecuarias \\ (DACA), (2) División Académica de Ciencias de la salud (DACS), Av. Universidad s/n \\ Zona de la Cultura Col. Magisterial, Villahermosa, C.P. 86040, Tabasco-México. \\ (e-mail: macronutriente@hotmail.com, (*) sabina52@hotmail.com, jblecastillo@hotmail.com, \\ corzososa@yahoo.com.mx)
}

* Autor a quien debe ser dirigida la correspondencia

Recibido Jul. 15, 2011; Aceptado Sep. 27, 2011; Versión Final recibida Oct. 15, 2011

\begin{abstract}
Resumen
El objetivo de este trabajo fue determinar el índice glucémico y la respuesta insulínica de una sopa conteniendo almidón nativo de banano. Los efectos benéficos del consumo de almidón resistente sobre el control glucémico han sido reportados previamente, pero estos parámetros no han sido suficientemente estudiados. Las determinaciones se realizaron en 12 sujetos sanos y 12 con diabetes tipo 2 y el índice glucémico de la sopa fue de 31.79. Las áreas bajo las curvas de glucosa e insulina fueron menores en los grupos con la sopa en comparación con el alimento de referencia $(\mathrm{P}<0.01)$. Sopa enriquecida con almidón nativo de banano podría ayudar a prevenir la aparición de diabetes tipo 2 en sujetos con alto riesgo y disminuir las complicaciones del diabético.
\end{abstract}

Palabras clave: almidón de banano, índice glicémico, índice insulinémico, almidón resistente

\section{Glycemic and Insulinic Response of Patients with Type 2 diabetes to the consumption of Pumpkin Soup Creole (Cucúrbita Pepo L.) Enriched with Banana Starch}

\begin{abstract}
The aim of this study was to determine the glycemic index value and the insulinic response of a soup containing native banana starch. The beneficial effects of resistant starch in dietary supplementation on glycemic control have been reported previously, but these parameters have not been sufficiently studied. Determinations were carried out in two groups: one of 12 healthy subjects and other with 12 type 2 diabetes. The estimated glycemic index value of the soup was 31.79. The total glucose and insulin areas under the curves were reduced in comparison with the reference food $(P<0.01)$. The enriched soup with native banana starch might help in preventing type 2 diabetes prevalence in health subjects with high risk. Also, this product can be used to decrease complications of people with diabetes.
\end{abstract}

Keywords: banana, glycemic index, resistant starch, insulin response, diabetes 


\section{INTRODUCCIÓN}

Una correcta dieta, tanto en individuos sanos como enfermos, es importante para lograr y mantener la salud, la integridad física y mental, obteniendo así una mejor calidad de vida. De esta forma, es importante conocer el efecto metabólico de los alimentos cuando se prescriben tratamientos nutricionales en forma de dieta oral, desde 1981 se desarrolló el concepto de índice glicémico de los alimentos, para clasificarlos de acuerdo con el efecto inmediato sobre los niveles de azúcar en sangre, ya que inicialmente se creía, que todos los alimentos con almidón en su estructura, producían el mismo índice glicémico y que debían ser omitidos de la dieta de pacientes diabéticos; se ha demostrado, que los carbohidratos presentan diferentes comportamientos en el cuerpo humano, independientemente de su composición, no así de su arreglo espacial (Paradas y Rozowsk, 2008), extendiendo así el uso del índice glicémico a las enfermedades crónico degenerativas, entre ellas la diabetes mellitus tipo 2, con el fin de incluir en la dieta aquellos alimentos que ingresan glucosa a la circulación sistémica, de una manera gradual y controlada (Alayón et al., 2008).

La diabetes es una enfermedad crónica que se caracteriza por la aparición de hiperglucemia por defectos en la secreción de insulina, disminución en su actividad o ambas situaciones a la vez. La diabetes mellitus tipo 2 es una de las patologías más frecuentemente diagnosticados en México y su prevalencia aumenta conforme se incrementa la edad de la persona. El tratamiento de un paciente diabético requiere combinar adecuadamente los aspectos médico y nutricional, para prevenir complicaciones posteriores que afectan su estado de salud y pueden conducirlo más rápido a la muerte (NOM-015-SSA2-1994). En México, la cultura alimentaria favorece el desarrollo de la enfermedad, como es el consumo de una cantidad excesiva de calorías provenientes de grasas, carbohidratos, y de productos industrializados que difícilmente se excluyen de la alimentación (Aguirre et al., 2006). Debido a la importancia de la alimentación en este tipo de pacientes, el objetivo de este trabajo fue determinar el índice glucémico y la respuesta insulínica de una sopa conteniendo almidón nativo de banana, con la finalidad de desarrollar un posible sustituto o apoyo a las fórmulas entérales.

\section{METODOLOGIA}

El banano Gran Enano (Musa $A A A$ spp) se obtuvo de la empacadora de banano Ganadera Agropecuaria "Las Mirandas", ubicada en el Km 44.5 de la carretera Villahermosa-Teapa. La obtención del almidón en la División Académica de Ciencias Agropecuarias de acuerdo al método reportado por Aparicio (2000). La calabaza, leche entera, aceite, sal, cebolla y consomé fueron adquiridos en el Mercado Municipal "Pino Suarez" y otros supermercados de la ciudad de Villahermosa Tabasco.

Para la obtención del almidón se realizaron tres muestreos, tomando cada vez al azar, un racimo de plátano verde $(10 \mathrm{Kg})$ cosechado por el productor a los noventa días de aparición de la floración (Reyes, 2000), los frutos, se pesaron lavaron, cortaron y molieron; para separar la fibra, se utilizan tamices del número 30,80 y 100 consecutivamente y agua a $40^{\circ} \mathrm{C}$ conteniendo $0.3 \%$ de ácido cítrico, la fibra retenida en el tamiz se eliminó y el filtrado obtenido se sedimento en refrigeración, separando el sobrenadante por decantación al día siguiente, la pasta se secó en estufa de aire forzado a $40^{\circ} \mathrm{C}$ por $24 \mathrm{~h}$, y se pulverizó en un molino de aspas por $1 \mathrm{~min}$. Al almidón de banano se le realizo análisis proximal por el método de A.O.A.C. (1984) y se determinó almidón resistente por el método del kit Megazyme (AOAC, 2002.02, AACC, 32-40) la glucosa liberada se midió a una longitud de onda de $510 \mathrm{~nm}$, calculando el almidón resistente mediante la Ecuación 1:

$\%$ Almidón Resistente $=$ Absorbancia $(\Delta \mathrm{E} X 100 / \mathrm{W}($ Humedad $) \times 90$

La sopa deshidratada se preparó con los siguientes componentes: calabaza, leche entera, almidón de banano, cebolla, consomé, aceite y sal, en las proporciones que se presentan en el Tabla 1 y de acuerdo a las recomendaciones para la determinación del IG reportadas por BelloPérez et al. (2009) para este tipo de producto: proteína $8.4 \mathrm{~g}$, grasa $13.5 \mathrm{~g}$ y almidón disponible 50g. La calabaza, se troceó, se molió, se secó, se pulverizo y peso para ser mezclado con el 
almidón de banano y los demás componentes. (Figura 1). Se analizó la composición proximal del producto formulado en la Universidad Tecnológica de Tabasco (UTTAB) tomando como referencia las Normas Mexicanas (NMX-F:083-1986, 607-2002, 608-2002, 615-2004 y 312-1978).

Tabla 1. Formulación de la sopa

\begin{tabular}{|lc|}
\hline & Cantidad \\
Calabaza & $12.00 \mathrm{~g}$ \\
Leche & $39.51 \mathrm{~g}$ \\
Almidón & $40,99 \mathrm{~g}$ \\
Aceite & $5.29 \mathrm{~g}$ \\
Consomé & $2.00 \mathrm{~g}$ \\
Cebolla & $0.80 \mathrm{~g}$ \\
Sal & $0.40 \mathrm{~g}$ \\
Total & $100 \mathrm{~g}$ \\
\hline
\end{tabular}

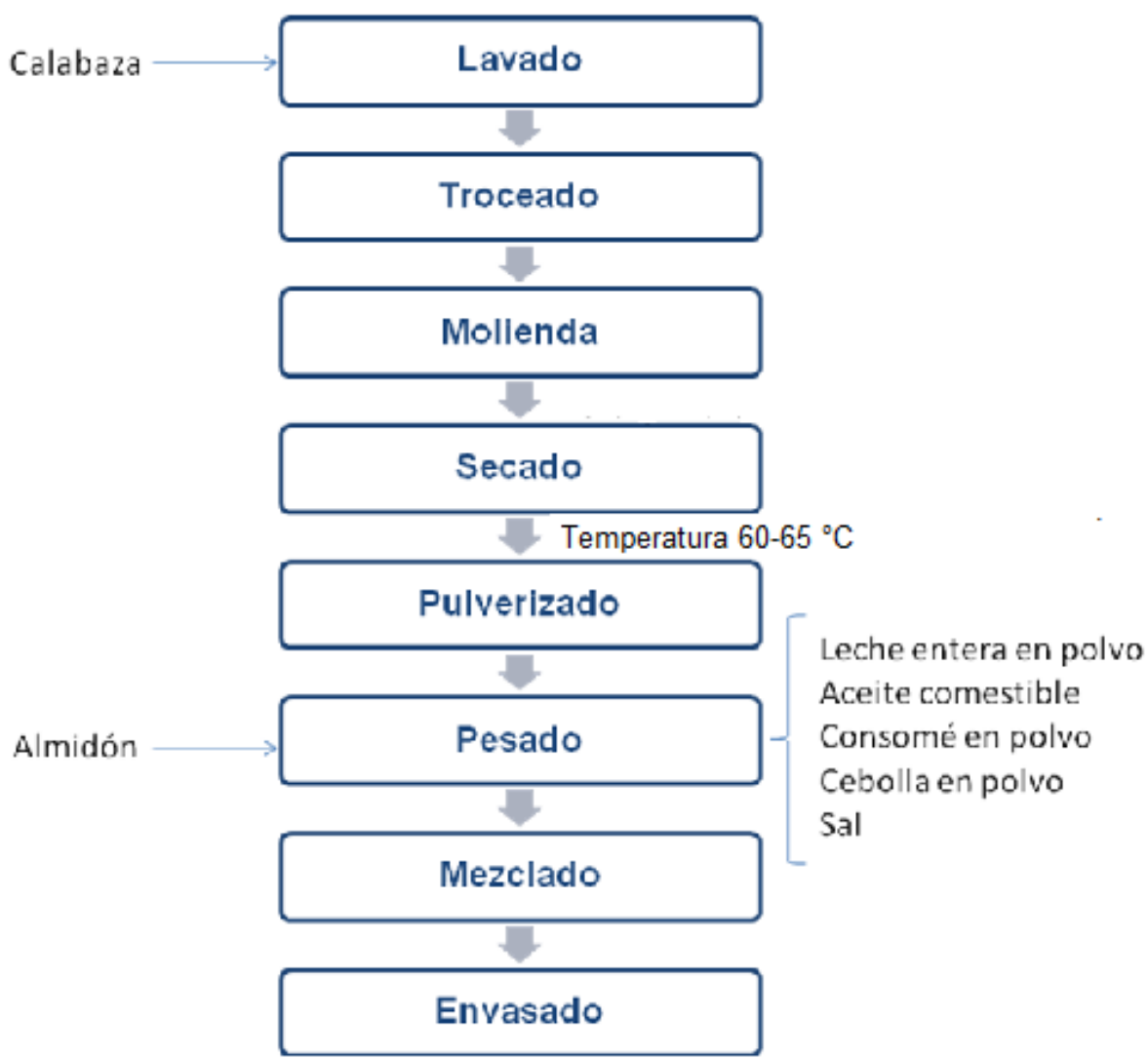

Fig. 1: Esquema de obtención de la sopa deshidratada de calabaza y almidón de banano

\section{Ensayo Clínico}

Las tablas internacionales del índice glicémico recomiendan utilizar de cinco a siete sujetos para determinar el índice glicémico de un alimento (Gretebeck et al., 2002), en el presente estudio participaron 12 sujetos voluntarios, cuyo procedimiento de selección se realizó de acuerdo a los principios éticos nacionales e internacionales para las investigaciones médicas en seres humanos (Declaración de Helsinki. Helsinki, 2000), para lo cual se invitó a los probables participantes, a una reunión en el Hospital de Zona No. 46 del Instituto Mexicano del Seguro Social (IMSS) donde se explicó el proyecto de investigación, los objetivos, procedimientos y peligros potenciales del 
ensayo, formando dos grupos de estudio de ambos sexos según los datos personales: edad, sexo, peso y talla, antecedentes familiares, tiempo de haber sido diagnosticado la enfermedad y tipo de tratamiento terapéutico en el caso de pacientes diabéticos. Cada sujeto tuvo una consulta con el nutriólogo antes de consumir el alimento de prueba. Los sujetos sanos fueron instruidos a consumir una dieta moderadamente alta en hidratos de carbono (por lo menos $300 \mathrm{~g} / \mathrm{día}$ ) tres días antes de la prueba para homogenizar el consumo previo de nutrimentos e hidratos de carbono (Chan, 2001), se determinó su requerimiento calórico tomando en cuenta su talla, peso y sexo (Harris y Benedict, 2006; Bergman y Hill, 2000 y Casanueva, 2001), las Tablas 2 y 3 muestran la distribución de macronutrimentos según su energía, en 5 tiempos de comida.

Tabla 2. Distribución de nutrientes

\begin{tabular}{|l|c|c|c|}
\hline \multicolumn{1}{|c|}{ Nutrientes } & $\%$ & Gramos & Kilocalorías \\
\hline Hidratos de Carbono & 60 & 300 & 1200 \\
\hline Proteínas & 15 & 75 & 300 \\
\hline Lípidos & 25 & 56 & 500 \\
\hline Total & 100 & 431 & 2000 \\
\hline
\end{tabular}

Tabla 3. Distribución de nutrientes por tiempos de comida/Kilocalorías

\begin{tabular}{|l|c|c|c|c|c|}
\hline \multicolumn{1}{|c|}{ T. de Comida } & $\%$ & Energía & Glúcidos (Kcal) & Proteínas (Kcal) & Lípidos (Kcal) \\
\hline Desayuno & 20 & 400 & 240 & 60 & 100 \\
\hline C. Diurna & 10 & 200 & 120 & 30 & 50 \\
\hline Comida & 40 & 800 & 480 & 120 & 200 \\
\hline C. Vespertina & 10 & 200 & 120 & 30 & 50 \\
\hline Cena & 20 & 400 & 240 & 60 & 100 \\
\hline Total & 100 & 2000 & 1200 & 300 & 500 \\
\hline
\end{tabular}

Descripción de los grupos de estudio

Se conformó un grupo testigo y un grupo experimental con 12 sujetos cada uno. El grupo testigo consistió en sujetos sanos de ambos sexos, con edades entre 20 y 50 años, con un índice de masa corporal entre 25 y 30, mientras el grupo experimental consistió en los 12 pacientes seleccionados de ambos sexos, con edades entre 20 y 50 años, con fecha de diagnóstico de diabetes mellitus tipo 2 no mayor a 10 años, con índice de masa corporal entre 25 y 30 (Tabla 4), sin complicaciones renales o hepáticas activas, con hemoglobina glucosilada $<8 \%$, con glucemias no mayores a $180 \mathrm{mg} / \mathrm{dL}$ y con dieta o con antihipoglucemiantes orales como control glucémico (Gattás, 2007, Camarena, 2006, Granfeldt, and Björck, 2006, Pérez, 2006).

Tabla 4. Características e indicadores bioquímicos de los sujetos en los que se determinó el índice glicémico.

\begin{tabular}{|l|l|l|}
\hline Características & Controles & Diabéticos \\
\hline$N^{\circ}($ Masc. / Fem.) & $12(5 / 5)$ & $12(5 / 5)$ \\
\hline Edad (años) & $25,3 \pm 4$ & $46 \pm 3,4$ \\
\hline Peso $(\mathrm{kg})$ & $62,18 \pm 7$ & $70,50 \pm 7,6$ \\
\hline Talla $(\mathrm{cm})$ & $165 \pm 0,06$ & $166 \pm 0,07$ \\
\hline IMC $(\mathrm{kg} / \mathrm{m} 2)$ & $22,87 \pm 1,9$ & $25,75 \pm 2,5$ \\
\hline Glucosa $(\mathrm{mg} / \mathrm{dl})$ & $82,75 \pm 8,5$ & $160,83 \pm 7$ \\
\hline
\end{tabular}




\section{Tratamientos}

Las determinaciones de la respuesta glucémica se realizaron según el método FAO, (1997) para determinar el índice glucémico de los alimentos. En este procedimiento se aplicaron dos tratamientos (T1 y T2). Los voluntarios de ambos grupos de estudio, se presentaron en ayuno, con un mínimo de 12 horas después de haber ingerido su último alimento y se realizó una determinación de glucemia en ayunas en muestras de sangre venosa periférica. El tratamiento T1 consistió en la ingestión de $50 \mathrm{~g}$ de glucosa anhidra; los sujetos fueron instruidos para ingerir la totalidad de la porción ofrecida en un plazo de 15 minutos, permitiéndoseles solo 1 vaso de agua $(240 \mathrm{ml})$ como acompañante. A los $15 \mathrm{~min}$ de haberse iniciado la ingestión se tomó la segunda muestra de sangre y se repitió a los diferentes tiempos $0,15,30,45,60,90$ y 120 min. (Tabla 5) Posteriormente se graficaron los resultados de glucemia/tiempo. Cinco días después de realizado el tratamiento 1, se aplicó el tratamiento T2, que consistió en $75 \mathrm{~g}$ del alimento bajo estudio (sopa en polvo conteniendo AR) rehidratada con $240 \mathrm{ml}$ de agua, repitiendo los mismos tiempos y monitoreo que para el tratamiento 1.

Tabla 5. Aplicación de tratamientos

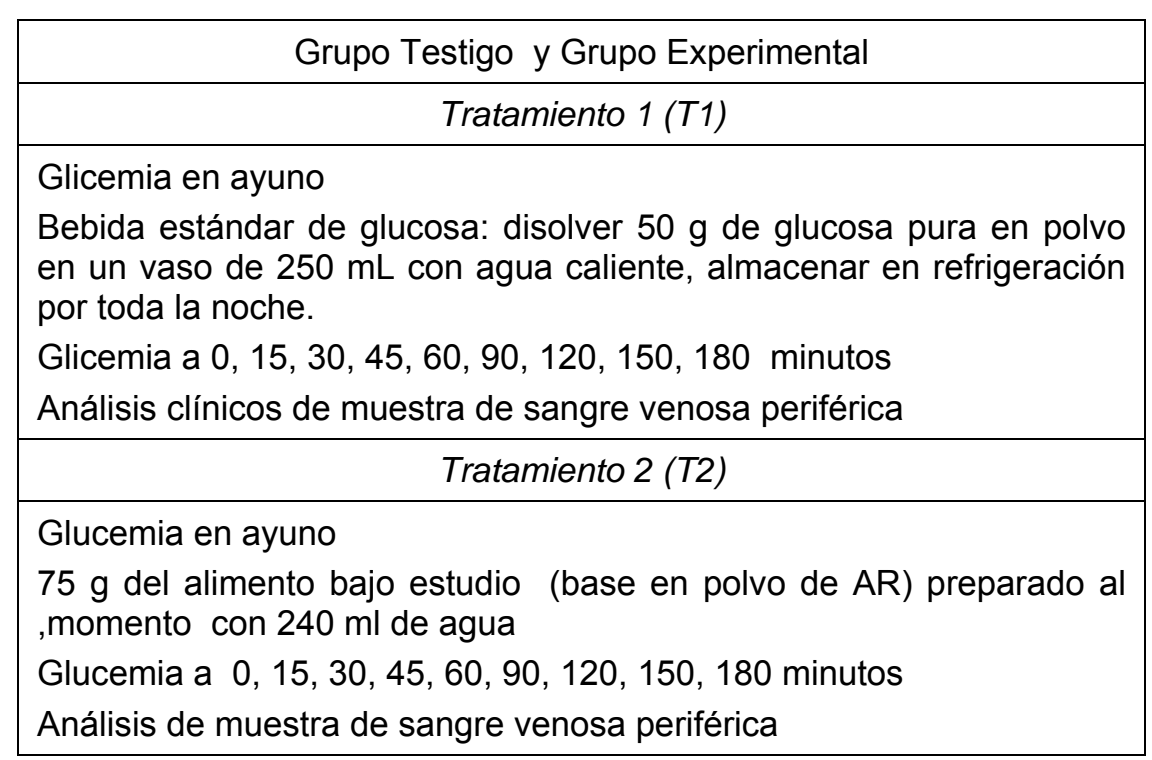

\section{Análisis clínicos}

Se recolectaron muestras de sangre periférica en ayunas y a los tiempos ya mencionados. Se separaron las muestras de suero por centrifugación, antes de la hora de su recolección y se conservaron en refrigerador a $-70^{\circ} \mathrm{C}$ hasta la realización de las diferentes pruebas. La determinación de glucosa, se realizó por medio del método enzimático-colorimétrico (Trinder, 1969) y por medio del autoanalizador SYNCHRON (Beckmann, 1997). Dichos equipos cuentan con un sistema de control de calidad continuo con sueros de control normal y anormal cada día. Los coeficientes de variación para cada prueba deben ser menores del $5 \%$.

Tanto para el tratamiento 1 (alimento de referencia) como para el tratamiento 2 (alimento en estudio) se graficó el promedio de los valores de la concentración de glucosa en sangre (glucemia), obtenidos en los análisis clínicos, y se calculó el área bajo la curva de la respuesta postprandial al alimento. El Índice Glicémico se calculó a partir del valor basal (glucemia en ayuno) por medio de la ecuación 2:

$I G=\frac{\text { Área bajo la curva del alimento a estudiar } \times 100}{\text { Área bajo la curva del alimento de referencia }}$

El valor encontrado en la ecuación 2 se dividió entre 1.4 para reportar los resultados tomando como base la glucosa (Leeds et al., 2004). Con el fin de conocer la respuesta metabólica de los 
alimentos en estudio, se calculó no solo el IG sino también el Índice Insulinémico (InIn), siguiendo los mismos pasos anteriormente descritos para el IG, pero utilizando los datos obtenidos de la concentración de insulina en las muestras de sangre en el análisis clínico.

\section{Análisis estadístico}

A los datos obtenidos se les aplico un análisis de varianza (ANOVA) de dos factores cuyas fuentes de variación fueron tiempo y glucosa para IG y tiempo insulina para InIn. La diferencia mínima significativa (DMS) se determinó con la prueba de Fisher $(\alpha \leq 0.05)$; los análisis se realizaron con el programa Graph-pad prism (versión 3.0, GraphPad Software, San Diego).

\section{RESULTADOS}

Se obtuvo un rendimiento de almidón/kilogramo de banano verde de $150 \pm 0.8 \mathrm{~g}$ con almidón resistente (AR) $34.22 \pm 1.5$ cuya composición química proximal se presenta en la Tabla 6 . La fórmula sin hidratar del alimento estudiado presentó valores promedio de $4.29 \pm 0,2 \%$ de humedad, $11.77 \pm 0,06, \%$ de proteína, grasa $12.47 \pm 0,07 \%$, carbohidratos $66.26 \pm 0,26 \%$ y $4.79 \pm 0,3 \%$ de cenizas.

Tabla 6. Composición química porcentual del almidón de banano

\begin{tabular}{|ll|}
\hline Humedad\% & $2.8 \pm 0,6$ \\
Proteínas\% & $2.3 \pm 0,1$ \\
Grasas\% & $2.7 \pm 0,3$ \\
Cenizas\% & $1.1 \pm 0.5$ \\
Carbohidratos & \\
(por diferencia) & $91.1 \pm 1.5$ \\
\hline
\end{tabular}

EI IG de la formulación a base de almidón de banano y calabaza fue de 31.79 según el cálculo de las áreas bajo la curva, el efecto del alimento de referencia en el grupo de sujetos sanos presenta un pico hiperglucémico a los 45 minutos con promedio $( \pm 134,74 \mathrm{mg} / \mathrm{dL})$, sin embargo cuando se administró la sopa a base de calabaza y almidón de banano, el pico hiperglucémico se redujo a niveles normales promedio $( \pm 96,67 \mathrm{mg} / \mathrm{dL})$. En el caso de los pacientes diabéticos los picos hiperglucèmicos se alcanzan a los 45 minutos para los dos alimentos, pero con valores mayores para el alimento de referencia.

\section{DISCUSION}

El rendimiento del almidón fue mayor que el obtenido por Reyes (2000), Gorosquer, et al, (2004) y Rivas-González et al., (2008), pero menor que el obtenido por Pérez, (2006), esto pudiera deberse tanto a las condiciones de procesamiento de la muestra como a la variedad de plátano, toda vez que los autores citados utilizaron plátanos verdes de la especie Musa sapientum $\mathrm{L}$. mientras que en este estudio se utilizó banano, Gran Enano (Musa AAA spp).

En cuanto al almidón resistente (AR) para el banano verde estudiado (Tabla 7), resultó mayor al reportado por Pérez (2006), pero similar a Lara (2010), mientras que en cereales como trigo y maíz ceroso (sin amilosa) se ha reportado $0.8 \%$ y $16 \%$ para un almidón alto en amilosa, que por tratamiento en autoclave aumentó a $5 \%, 12.4 \%$ y $18 \%$ respectivamente (Russel et al., 1988). La resistencia a la digestión de los almidones ha sido relacionada con alto contenido de amilosa; Reyes (2000) y Aparicio (2003) reportaron del 30-36 \% de amilosa en este mismo tipo de almidón, donde la resistencia a la digestión fue mayor, que para almidón de trigo y maíz ceroso, pero con comportamiento semejante a la reportada por Russel et al., (1988) para un almidón alto en amilosa. 
Tabla 7. Contenido de almidón resistente en plátano y banano

\begin{tabular}{|l|c|c|}
\hline \multicolumn{1}{|c|}{ Autor } & Producto & \% Almidón \\
\hline Pérez, (2006) & Almidón Plátano & 19.62 \\
\hline Lara, (2010) & Almidón Banano & Min 28. 77, max 35.18 \\
\hline En este trabajo & Almidón Banano & $34.22 \pm 1.5$ \\
\hline
\end{tabular}

Al comparar la composición proximal y el aporte energético de la sopa deshidratada de banano verde, con una bebida a base de almidón de plátano y con una sopa comercial a base de vegetales mixtos y almidón de maíz modificado, la fórmula sin hidratar del alimento estudiado (Tabla 8) ubica a este producto en un alimento energético principalmente por su contenido de hidratos de carbono ya que proporciona $319 \mathrm{Kcal} /$ porción de $75 \mathrm{~g}$. Esta característica se encuentra en la mayoría de las sopas comerciales de vegetales, donde las proporciones de las grasas y proteínas tienden a variar de acuerdo a los sólidos presentes, pero siempre con una mayor proporción de carbohidratos, seguido de la proteína y las grasas, no solo contribuyen a la saciedad del hambre, sino también al balance nutricional energético y tienen efecto positivo sobre algunas funciones biológicas del organismo humano por lo que se considera, que la ingesta de este tipo de alimento, podría favorecer el mantenimiento del balance de nutrientes en la dieta. (Wicks et al., 2006).

Por su contenido de humedad el alimento estudiado, es un producto estable, que puede conservarse durante largos periodos de tiempo, presentando valores menores a los establecidos por las normas de calidad en México (NMX-F-083-1986 y NMX-F-058-1968), según las cuales en mezclas deshidratadas de caldos y sopas el contenido de humedad no debe ser mayor de $10 \%$.

Tabla 8. Composición química y calórica por $100 \mathrm{~g}$ y por porción.

\begin{tabular}{|l|l|l|}
\hline Parámetros & Por $100 \mathrm{~g}$ & Por porción $(75 \mathrm{~g})$ \\
\hline Energía & $425.3 \pm 0,05 \mathrm{Kcal}$. & $319 \pm 0,05 \mathrm{Kcal}$. \\
\hline Proteína & $11.77 \pm 0,06 \%$ & $8.28 \pm 0,06 \%$ \\
\hline Grasas & $12.47 \pm 0,07 \%$ & $9.35 \pm 0,07 \%$ \\
\hline Carbohidratos & $66.26 \pm 0,26 \%$ & $49.7 \pm 0,26 \%$ \\
\hline Humedad & $04.29 \pm 0,2 \%$ & $3.21 \pm 0,2 \%$ \\
\hline Cenizas & $04.79 \pm 0,3 \%$ & $3.59 \pm 0,3 \%$ \\
\hline
\end{tabular}

Tomando en cuenta que la porción utilizada en la preparación de la sopa es de $75 \mathrm{~g}$ y que tanto las proteínas como la grasa son proporcionadas por la leche entera, estos nutrientes resultaron semejantes que los reportados en el contenido nutricional de los productos comerciales. En términos de energía la formulación en estudio está dentro los valores nutrimentales de referencia, ya que es similar a otros productos en el mercado, dando una densidad calórica de 319 Kcal., de las cuales el $12 \%(10-15 \%)$ proviene de proteínas, $26 \%(25-30 \%)$ de grasas y $62 \%(60-65 \%)$ de hidratos de carbono (Fomento de nutrición y salud A. C. y INCMNSZ, 2009; Bourgues et al., 2004; FNM/IOM, 2002). Desde otro enfoque, se probó que la sopa aporta almidón resistente de banano por su resistencia a la hidrólisis con amilasas digestivas in vivo, posiblemente la resistencia intrínseca de los gránulos de almidón del banano verde, está relacionada a su proporción de amilosa/amilopectina (Faisant et al., 1995, Goñi et al., 1996, ) y a las condiciones de procesamiento térmico (Villada et al., 2008) que al no afectar su estructura nativa, permite clasificarlo como almidón resistente tipo II (Pacheco et al., 1998). 
Actualmente existe un considerable interés sobre el significado nutricional de las formas resistentes de los almidones en los alimentos (Yue y Waring, 1998), ya que diversas investigaciones sugieren, que alimentos de digestión lenta, con un IG bajo, en la dieta de individuos sanos y pacientes con diabetes e hipolipidemia, están asociados con la reducción de la respuesta glicemia y reducción de la secreción de insulina y lípidos en la sangre (Anderson et al., 1994; Granfeldt et al., 1995; Wolever y Brand, 1995); bajo este enfoque, la disponibilidad de AR en la sopa de banano es importante no solo por estar asociado a la fibra dietética, sino por las implicaciones sobre la tasa de hidrólisis de su almidón, que tiene influencia en la disminución de la curva postprandial presentando IG bajo (Pacheco y Testa, 2005; Pacheco, 2001).

Por otro lado, las formulaciones existentes en el mercado cuya fuente principal de hidratos de carbono son los almidones de maíz y/o maltodrextrinas contienen también sacarosa, provocando esta última osmolaridad alta (Pérez, 2006). Bebidas libres de lactosa y gluten que no contienen ningún tipo de sustancias conservadoras contienen canola y ácidos grasos omega-3: bajas en grasas saturadas y colesterol, son ricas en antioxidantes y proponen un sistema show release, que ayuda a que se tenga baja respuesta glucémica, contribuyendo a un mejor control de la glucosa sérica, además de prevenir la hiperglucemia postprandial (Aguirre et al., 2006); el único inconveniente de estos productos en el mercado es el costo en que se encuentran $\$ 48.00 / 240 \mathrm{~mL}$ (en pesos mexicanos) lo cual dificulta la adquisición por la población a la que están dirigidos. Además se exige que se especifiquen claramente los ingredientes y la distribución calórica del producto ya que se puede provocar complicaciones metabólicas por consumirlos indiscriminadamente o sin control (Pacheco, 2001).

\section{Respuesta glicémica e insulínica.}

EI IG 31.79 de la formulación a base de almidón de banano y calabaza en sujetos sanos, ha resultado de tomar como referencia la glucosa oral al 50\% (Figura 2) y de acuerdo con Leeds et al., (2004) se asigna el valor de 100 a el IG de la glucosa donde valores $\geq 70$ el IG es alto, 56-69 IG moderado y $\leq 55$ el IG es bajo, por lo que el alimento de prueba se encuentra en el rango de IG bajo, además por su almidón resistente a la digestión, la fórmula presenta ventaja doble al ser utilizada, ya que además de disminuir la respuesta postprandial, causó una sensación de plenitud mayor a la observada con el alimento de referencia al presentar los sujetos, menor ansiedad de ingesta de alimentos después del consumo de la sopa, que al consumir el alimento de referencia.

En la Figura 2 se observa un pico hiperglucémico a los 45 minutos con promedio $( \pm 134,74 \mathrm{mg} / \mathrm{dL})$ para el alimento de referencia, sin embargo cuando se administra la sopa a base de calabaza y almidón de banano el pico hiperglucémico aparece a los $30 \mathrm{~min}$ con 100,67 mg/dL reduciéndose a los niveles normales promedio $( \pm 96,67 \mathrm{mg} / \mathrm{dL})$ en $45 \mathrm{~min}$, esto es importante porque de esta estabilidad depende el aporte adecuado de glucosa al cerebro que utiliza el $50 \%$ en condiciones basales, y entre más baja sea la absorción de glucosa habrá menor secreción de insulina.

Como se observa en la Tabla 9, la respuesta a la liberación de insulina postprandial en los pacientes diabéticos, al consumir el alimento de referencia es mayor a los 90 min (28 unidades), que la respuesta al consumir la sopa (21 unidades) ya que persiste la hiperinsulinemia después de la ingesta de alimento de referencia mientras que la hiperinsulinemia en la formulación de almidón de banano y calabaza fue menor

En la Figura 3 se puede observar el efecto de la sopa sobre la respuesta insulínica en sujetos sanos, presentando una reducción en la respuesta insulínica a los 30 minutos cuando se emplea la sopa, comparada con el alimento de referencia. Como podemos observar en la Figura 4, en los pacientes con diabetes, el pico hiperglucémico es alcanzado a los $45 \mathrm{~min}$, igual que con el grupo de sujetos sanos de la Figura 2, pero el uso de la sopa reduce la hiperglucemia, lo cual se aprecia por el aplanamiento de la curva. 


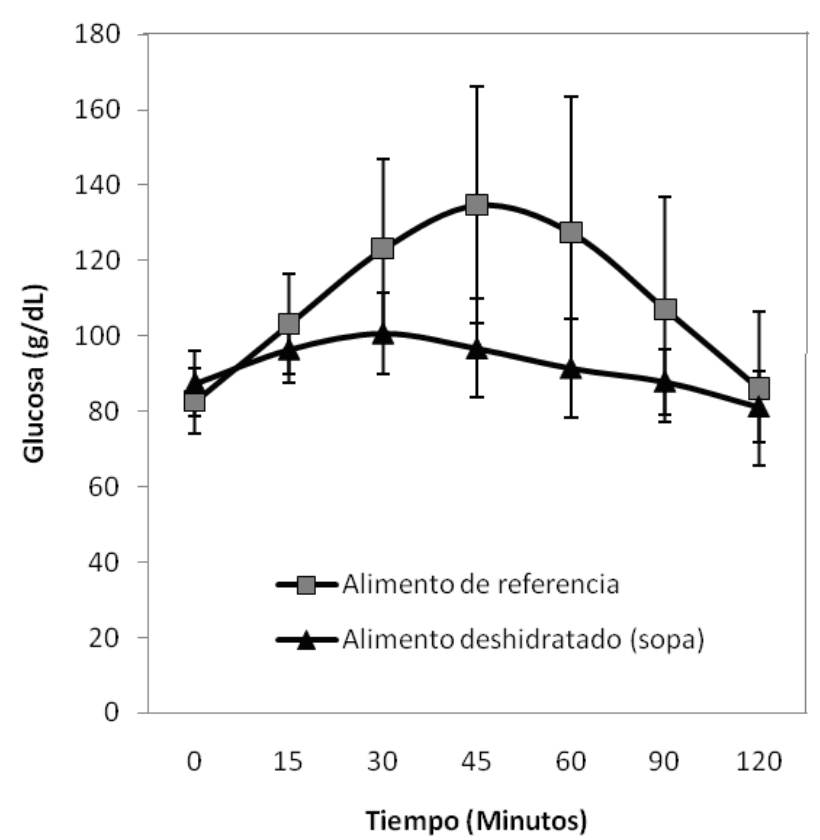

Fig. 2: Efecto de la sopa con calabaza y almidón de banano y del alimento de referencia (Glucosa oral) sobre los niveles de glucosa en sangre de sujetos sanos

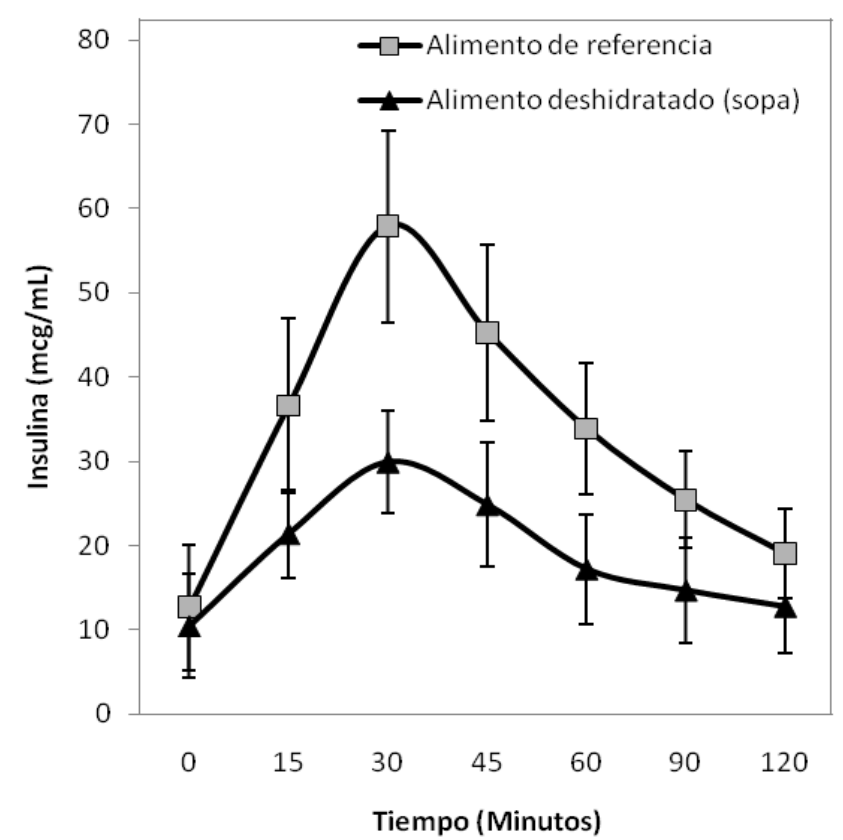

Fig. 3. Efecto de la sopa con calabaza y almidón de banano y del alimento de referencia (Glucosa oral) sobre los niveles de insulina en sangre de sujetos sanos

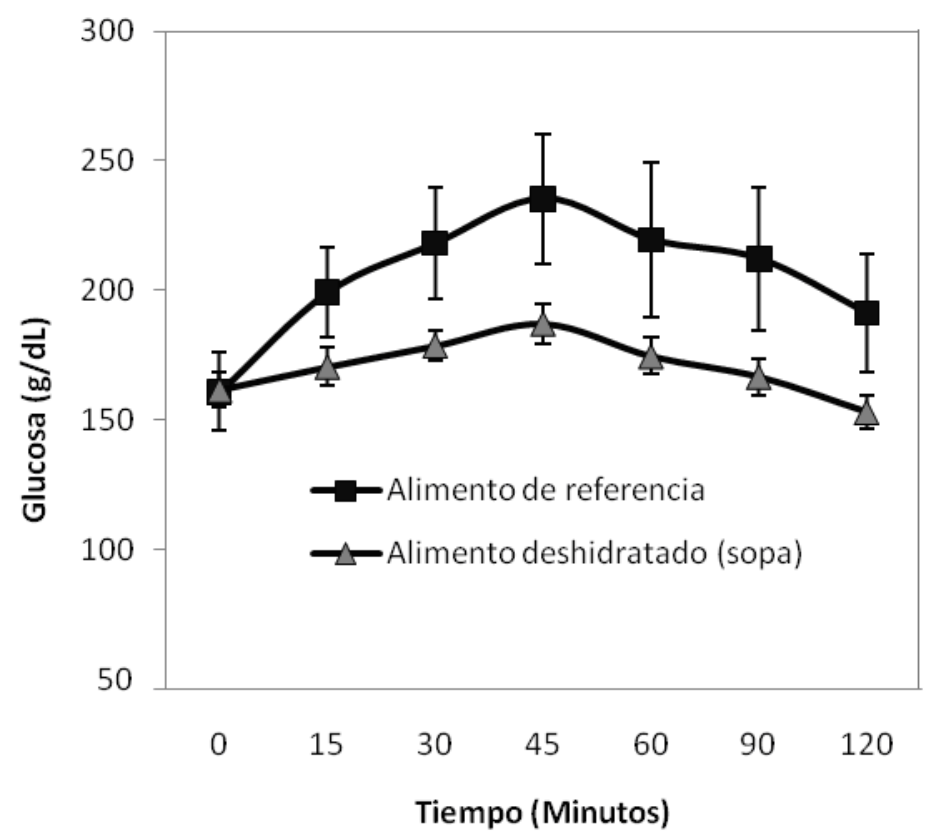

Fig. 4: Efecto de la sopa a base de almidón de banano y calabaza sobre los niveles de glucosa en sangre de pacientes diabéticos comparados con la respuesta del alimento de referencia (Glucosa oral)

La Asociación Americana de Diabetes (ADA) establece que el tratamiento de la DM incluye de preferencia el empleo de carbohidratos complejos, alimentos con alto contenido de fibra y bajos en grasa y un consumo moderado de sodio. El alimento con almidón de banano entra dentro de la referencia, además de que al disminuir las posibles complicaciones crónicas microvasculares, el tratamiento que reciben estos pacientes es mejorado ya que las cifras alcanzadas $(235 \mathrm{mg} / \mathrm{dL})$ para el alimento de referencia (Tabla 9), cuando la norma establece $140 \mathrm{mg} / \mathrm{dL}$ postprandial, son consecuencia tanto de la producción hepática excesiva, como de la falta de utilización de glucosa en músculo, la hiperglucemia en parte es responsable de disminuir la respuesta de la célula $\beta$ al 
estímulo de la glucosa y de inducir y mantener alterada la acción de insulina, por lo anterior, la hiperglucemia además de ser un marcador bioquímico, es un factor patogénico, que perpetúa el estado diabético, por eso ese efecto de tóxico de glucosa, implica que cualquier medida que disminuya la hiperglucemia como por ejemplo alimentos que tengan un factor hipoglucemiante incluso insulinémico, aumentará los niveles circulantes de la hormona y mejorara su acción y la sopa presenta una respuesta lenta $(187 \mathrm{mg} / \mathrm{dL})$ siendo su inicio de $161 \mathrm{mg} / \mathrm{dL}$ en un espacio de 1 hora aumentando solo $26 \mathrm{mg} / \mathrm{dL}$ en comparación con el alimento de referencia.

Tabla 9. Efecto del alimento de referencia (Glucosa oral) y la sopa a base de almidón de banano y calabaza en la liberación de insulina en pacientes diabéticos.

\begin{tabular}{|c|c|c|c|c|}
\hline Insulina $\mathrm{mcg} / \mathrm{mL}$ en DM & \multicolumn{2}{|c|}{ Alimento de referencia } & \multicolumn{2}{c|}{ Sopa } \\
\hline $\mathrm{T}(\mathrm{Min})$ & Promedio & $\mathrm{N}$ & 14 & $\mathrm{~N}$ \\
\hline 0 & 15 & 12 & 20 & 12 \\
\hline 15 & 17 & 12 & 27 & 12 \\
\hline 30 & 22 & 12 & 24 & 12 \\
\hline 45 & 25 & 12 & 22 & 12 \\
\hline 60 & 30 & 12 & 21 & 12 \\
\hline 90 & 28 & 12 & 18 & 12 \\
\hline 120 & 23 & 12 & & \\
\hline
\end{tabular}

Además de que el alimento de prueba no eleva los niveles de glucosa postprandial arriba de 200 $\mathrm{mg} / \mathrm{dL}$, la digestión se presenta en forma lenta y gradual debido a su bajo índice glucémico y con respecto a la respuesta insulínica aumenta la sensibilidad en 8-10 min (Wartofsky, 2004) alcanzando su concentración pico en sangre periférica a los $30-45$ minutos, seguida de un rápido descenso de la glucemia postprandial, que regresa a los niveles previos después de 90-120 min. De los nutrientes que normalmente se ingieren, la glucosa es la que más estimula la secreción de insulina y en menor cantidad las proteínas y después los ácidos grasos libres (Torres y Herrera, 2005). En el grupo de los sujetos sanos se alcanzan respuestas más rápidas de 57.97 para el alimento de referencia y 29.95 para la sopa porque su reserva pancreática esta normal, pero si se insiste en consumir alimentos de fácil digestión la reserva disminuye con el tiempo.

Una respuesta lenta a la digestión de hidratos de carbono permite al organismo regular de manera gradual la absorción de los nutrientes y las alteraciones metabólicas disminuyen (Korenman y Heber, 2006), la respuesta de insulina que ocurre en forma aguda en los primeros 30 min posteriores a la ingesta del alimento (de prueba y de base) es insulina preformada, es decir la almacenada dentro de los gránulos de las células $\beta$ y se presenta en la circulación como un pico con una duración de 15-30 min; si el estímulo persiste, la concentración de insulina se eleva nuevamente; como segunda fase de secreción o fase tardía, por otra parte si la glucemia se mantiene elevada por mucho tiempo, las células $\beta$ se desensibilizan a este estímulo, pero no a otros, es decir ya no responden a glucosa pero si a otros nutrientes tales como amino ácidos y ácidos grasos libres provocando la producción de triglicéridos en el hígado (Gattás, 2007).

La utilización corporal de la glucosa después de su ingesta, ocurre en tejidos independientes de insulina: cerebro $50 \%$, hígado e intestino (25\%) y el $25 \%$ restante se lleva a cabo en los tejidos dependiente, principalmente en el músculo (Flores, et al., 2001). Para que se pueda mantener este equilibrio, depende de que ocurra en forma simultánea y coordinada los siguientes procesos: secreción apropiada de insulina y captación adecuada de glucosa por el músculo (tejido periférico dependientes) hígado e intestino (tejidos esplácnicos) (Torres y Herrera, 2005). 
El tránsito de un alimento por el tracto digestivo es de 6-8 horas y su permanencia en el colon puede ser de hasta 2-3 días (Korenman y Heber, 2006), si bien la resistencia del alimento es cuantitativamente un componente minoritario en las alimentación humana, su paso a través del estómago y el intestino delgado, sin sufrir digestión ni absorción, explicaría que en el colon el almidón de banano contenido en la sopa, se puede comportar como un material más presentes en el mismo, y puede desarrollar los efectos fisiológicos siguientes: aumento del peso y disminución de la consistencia de la heces, disminución del tiempo del tránsito intestinal debido al contenido de almidón de banano encontrado (34.22\%) por lo que se recomienda sea objeto de un trabajo posterior que este enfoque.

En las Figuras 2, 3, 4, y 5 se observa el desarrollo de la digestión in Vivo del alimento conteniendo almidón de banano con respecto al tiempo y se confirma lo publicado por investigadores como Eggleston, et al., (1992), quienes afirman que el almidón de plátano verde es resistente a las amilasas pancreáticas. Esta resistencia a la degradación podría atribuirse a varios factores físicos tales como la morfología y estructura del gránulo (Paradas y Rozowskin, 2008), el almidón de plátano posee formas alongadas con diámetros de $40 \mu \mathrm{m}$ y longitud hasta de $51.7 \mu \mathrm{m}$ (Aparicio, 2003); Leeds et al., (2004) mencionan que la respuesta fisiológica a los alimentos que contienen diferentes tipos de carbohidratos presenta también diferentes efectos en el metabolismo humano debido a las diferentes tasas de digestión y a la velocidad de absorción

En las Figuras 5 y 6 se comparan los efectos entre el alimento de referencia y la fórmula y entre los dos grupos en estudio (sanos y diabéticos), previsiblemente, los niveles de glucemia son más elevados en el punto basal en pacientes diabéticos que en los sujetos sanos, los picos hiperglucémicos se presentan a tiempos diferentes y la disminución en la glucemia e insulinemia postprandial en los sujetos sanos, es significativa $(p<0.001)$ con respecto a los pacientes diabéticos, a los 120 minutos como puede observarse en las Figuras 7 y 8 . Además la insulina es una hormona no solo involucrada en la regulación de glucemia, si no también desempeña papel importante en cuándo y cómo se almacena grasa, ya que a menudo existen altos niveles de insulina en las personas obesas, o con altos niveles de grasa en la sangre, ya sea colesterol o triglicéridos (Korenman y Heber, 2006).

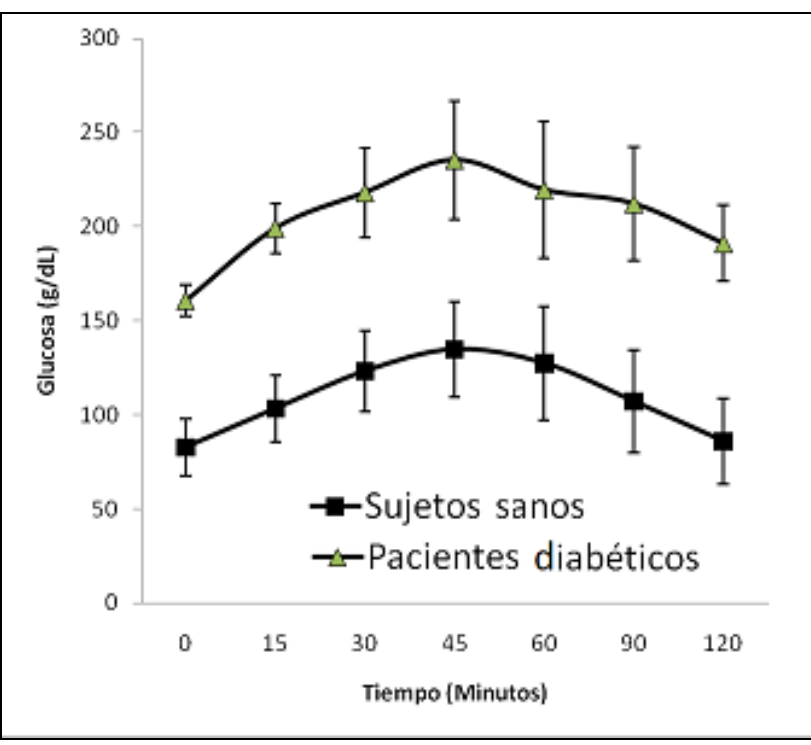

Fig. 5: Comparación de los niveles de glucosa en sangre a través del tiempo en sujetos sanos y pacientes diabéticos después de ingerir el alimento de referencia (Glucosa Oral)

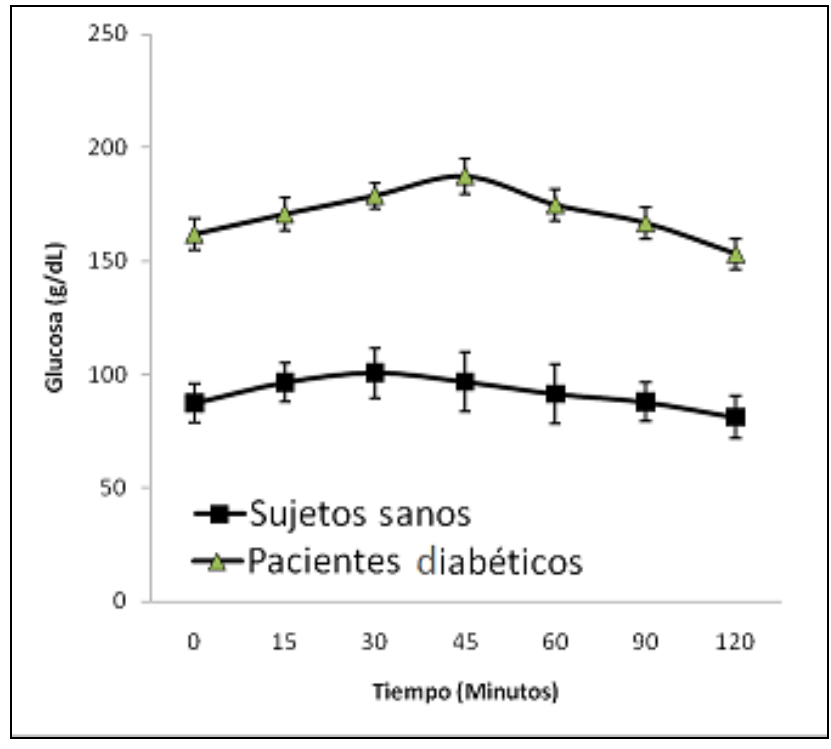

Fig. 6: Comparación de los niveles de glucosa en sangre a través del tiempo en sujetos sanos y pacientes diabéticos después de ingerir el alimento con almidón de banano y calabaza. 


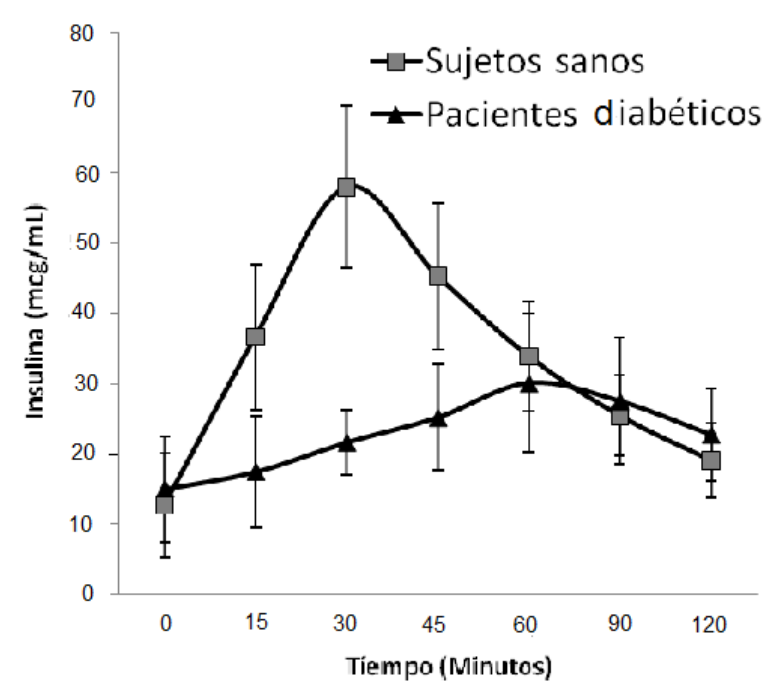

Fig. 7: Respuesta insulínica a través del tiempo en sujetos sanos y pacientes diabéticos después de ingerir el alimento de referencia (Glucosa Oral).

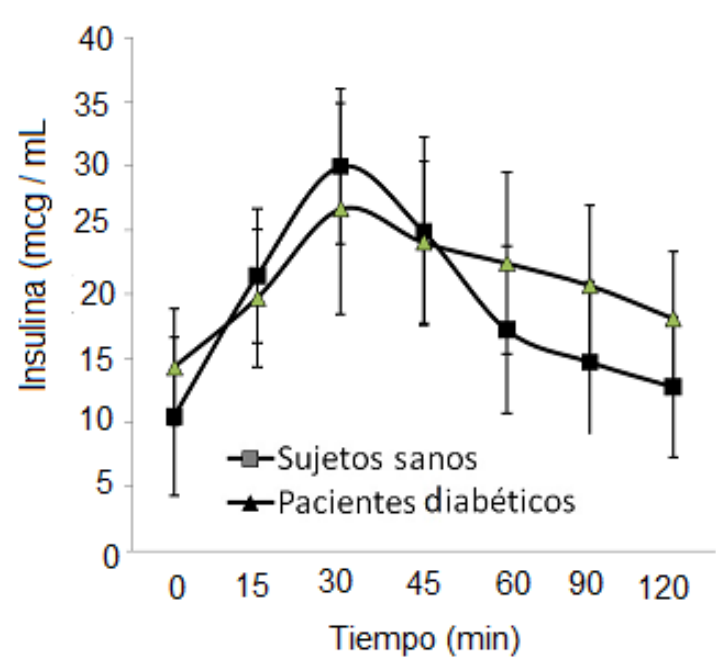

Fig. 8. Respuesta insulínica a través del tiempo en sujetos sanos y pacientes diabéticos después de ingerir el alimento con almidón de banano y calabaza.

El estudio de la hidrólisis del almidón in Vitro permite obtener información relativa sobre el grado de gelatinización en un producto y predecir la biodisponibilidad del almidón (Tovar, 2001) cuanto menos gelatinizado (hinchado) esté el almidón, más lenta será la velocidad de digestión (Leeds et al., 2004) el resultado obtenido (34.22\%), ubica al almidón de banano verde como una fuente de carbohidratos de digestión lenta.

La evidencia presentada hace posible que los cristales de amilosa sean responsables del AR, pero no establece de manera inequívoca cuándo se generan estos cristales. Actualmente la identificación del AR, involucra una digestión con enzima amilolítica; por tanto, sigue siendo posible que el AR sea generado durante la incubación es decir, los $\alpha$ - glucanos cortos $(1 \rightarrow 4)$ podrían cristalizar como moléculas separadas, movilizadas por el rompimiento de la amilosa, más que por los segmentos movilizados térmicamente dentro de las macromoléculas de amilosa. Este último mecanismo podría quizás ser probado mediante el desarrollo de métodos para detectar y medir los cristales de amilosa dentro del gránulo de almidón antes de la amilólisis. El primer mecanismo es más complejo, y por lo tanto menos atractivo, debido a que este implica la existencia de un estado extra potencialmente resistente de la amilosa (Rusell et al., 1988).

\section{CONCLUSIONES}

El almidón de banano por sus propiedades funcionales, composición fisicoquímica y baja digestibilidad, puede ser utilizado en formulaciones de harinas compuestas o como ingrediente principal en la preparación de polvos de mezclas para sopas instantáneas, e incluso como aditivo natural en la formulación de nuevos productos. Por su aporte nutricional (bajas en grasa, alto porcentaje de fibra dietética y almidón resistente) las sopas deshidratadas de harina de banano verde y calabaza, pueden ser recomendadas para regímenes especiales de alimentación dado su índice glicémico bajo, sobre todo cuando exista la predisposición genética a ser diabético,

\section{AGRADECIMIENTOS}

Los autores agradecen al Hospital de Zona No. 46 del Instituto Mexicano del Seguro Social (IMSS) a la empacadora de banano Ganadera Agropecuaria "Las Mirandas", a la Unión Agrícola Regional de la Sierra del Estado de Tabasco productora de Plátano y a la Fundación Produce Tabasco A.C. por el apoyo otorgado para la realización de este trabajo 


\section{REFERENCIAS}

A.A.C.C. Approved methods of the AACC. American Association of Cereal Chemist, St. Paul, MN. 23-25 (2002).

A.O.A.C. Official Methods of Analysis. Association of Official Analytical Chemists. Washington, D.C., USA. Method 2002.02. 35-39 (2002).

A.O.A.C. Official methods of analysis of the association of official agricultural chemists. 10 ed. Washington, EEUU. 17-19 (1984).

Aguirre, P., Galgani F., Díaz B. Determinación del índice glicémico del alimento NUTRIDIABETIC® destinado a diabéticos tipo 2. Rev. Chil. nutr., v. 33. Santiago de Chile, 4-6 (2006).

Alayón, A., Mosquera V., Alvear S. Control glucémico y metabólico integral: dos metas complementarias para el paciente diabético, Rev. Salud Uninorte. Barranquilla Vol. 24 (2), 8-9 (2008).

Anderson, J. W., Smith, B. M. y Gustafson, N. J. Health benefits and practical aspects of high fiber diets. Am. J. Clin Nutr. 55: 12425 - 12475 (1994).

Aparicio, T. M. A., Comportamiento fisicoquímico y reológico de los almidones nativos y modificados de yuca, camote y plátano macho. Reporte técnico. CONACYT-SIGOLFO. México, 19-23 (2000).

Aparicio, T. M., Caracterización fisicoquímica de los almidones nativos y modificados de yuca, camote y plátano Valery. Tesis doctoral. Instituto Tecnológico de Veracruz. Veracruz, México. 3649 (2003).

Bello-Pérez. L. A., Betancourt, D., y Bravo, L. Manual de métodos de caracterización de carbohidratos. pp. 76-91, México (1999).

Bello-Pérez, L. A., Agama-Acevedo, E., Sánchez-Hernández, L. y Paredes-López, O. Isolation and partial characterization of banana starches. Journal of Agricultural and Food Chemistry. 47, pp. 854-857 (1999).

Beckmann, T. Catálogo de autoanalizadores SYNCHRON 33410. 65-89 (1997).

Bergman, B.C. y Hill J. Thermogenesis. En: Guyton AC, Hall JE. Tratado de Fisiología Médica. pp. 757 - 772, 10a Ed. Madrid: McGraw- Hill Interamericana (2000).

Bourges, H., Casanueva, G., Rosado, J. Recomendaciones de ingestión de nutrimentos para población mexicana. Bases fisiológicas I. Vitaminas y Nutrimentos inorgánicos. pp 34-36, Ed. Médica Panamericana. México (2004).

Camarena, A. E., Determinación del Índice Glucémico de los Alimentos utilizando Cálculo Integral, Instituto de Ciencias Agrícolas (ICA), Universidad de Guanajuato. Guanajuato, México. 6-11 (2006).

Casanueva, E., Nutriología Médica. 356-364, 2da Ed. Edit. Panamericana. México (2001).

Chan, N., The glycaemic index values of Vietnamese foods. Eur. J. Clin. Nutr. 55: 1076-1083. (2001).

Declaración de Helsinki. Helsinki, Finlandia. Ratificada por la $52^{\circ}$ Asamblea General, Edimburgo, Escocia. 34-67 (2000).

Eggleston, G. I., Swennen, H. R. y Akoni, S. I. Physicochemical Studies on Starches Isolated from Plantain Cultivars, Plantain Hybrids and Cooking Bananas. Starch 44: 121-128. (1992).

Faisant, N., Gallant, D. J., Bouchet, B. y Champ, M. Banana starch breakdown in the human small intestine studied by electron microscopy. Eur. J. Clin. Nutr. 49: 98-104 (1995). 
FAO. Carbohydrates in human nutrition (FAO Food and Nutrition paper-66) Chapter 4 . The role of the glycemic index in food choice. FAO/WHO, Rome. 89-102 (1997).

Flores, F., Cabeza, A. y Calarlo, E. Endocrinología. pp 391-482. Ed. Méndez. México (2001).

FNB/IOM. Food and Nutrition Board/Institute of Medicine Dietery reference intakes for energy, Carbohydrate, Fiber, Fatty Acids, Cholesterol, Protein, and Amino Acids. pp. 465-608. (2002).

Fomento de nutrición y salud A.C y INCMNZ Instituto Nacional de Ciencias Medicas y Nutrición Salvador Subirán, Menú familiar para comer sanamente, 29-33, México (2009).

Gattás, V. Determinación de los índices glicémicos y de insulina en fórmulas para alimentación enteral en adultos sanos, Rev Méd Chile. 5-10 (2007).

Goñi, I., García-Diaz, L., Mañas, E., y Saura-Calixto, A. Analysis of resistant starch: a method for foods and food products. Food Chem. 56: 445-449 (1996).

Granfeldt, X. W. y Björck, I. Determination of glycaemic index; some methodological aspects related to the analysis of carbohydrate load and characteristics of the previous evening meal, Department of Applied Nutrition and Food Chemistry, Department of Food Technology, Engineering and Nutrition, Lund University, Sweden. 76-83 (2006).

Granfeldt, Y., Drews, A. y Drews A., Arepas made from high amylose corn flour produce favorably how glucose and insulin responses in healthy humans. J. Nutr. 123: 1676- 1684. (1995).

Gretebeck, R. J., Gretebeck K. A., Tittelbach T. J. Glycemic index of popular sport drinks and energy foods. JADA; 102: 415-417. (2002).

Harris, J. A. y Benedict, F. G. A. Biometric study of human basal metabolism.Proceedings of de National Academy of Sciences of the united States of America. 1918; 4; 370-373 (2006).

Korenman, S. y Heber, D. Atlas de Endocrinología Clínica, Nutrición y Obesidad Humana. Pp 421422, Ed. T. G. Hostench, S. A. España (2006).

Leeds, A Brand, M, J. y Powell, F. No todos los Carbohidratos engordan. pp. 59-60, Ed. Panorama. México (2004).

NMX-F-058. Alimentos. Sopas Deshidratadas. Normas Mexicanas. Dirección General de Normas. 3-9, México. (1968).

NMX-F-083. Alimentos. Determinación de Humedad en productos alimenticios. 4-12, México. (1986)

NMX-F-312. Alimentos. Determinación de reductores directos y totales en alimentos. 3-7, México. (1978).

NMX-F-607. Alimentos. Determinación de Cenizas en productos alimenticios. 5-16, México. (2002)

NMX-F-608. Alimentos. Determinación de Proteínas en alimentos. Métodos de prueba. 2-14, México. (2002).

NMX-F-615. Alimentos. Determinación de Extracto etereo (Método Soxhlet) en alimentos. Método de prueba. CANCELA A LA NMX-F-089-S-1978). 4-13, México. (2004).

NOM-015-SSA2. Norma Oficial Mexicana, Para la prevención, tratamiento y control de la diabetes mellitus en la atención primaria. 7-21, México. (1994)

Pacheco, D. E. Evaluación nutricional de sopas deshidratadas a base de harina de plátano verde, Digestibilidad in vitro del almidón. Acta Científica Venezolana, Venezuela 52, 278-282 (2001).

Pacheco, D. E., Sequera, B. y Herrera, I. Plant starches and oils. Their influence on digestión in rats. J. Sci. Food Agric. 77: 381-386 (1998) 
Pacheco, E. y Testa G. Evaluación nutricional, física y sensorial de panes de trigo y plátano verde. Revista Interciencia 30(5), 300-305 (2005).

Paradas, S. J. A. y Rozowskin, J. Relación entre la respuesta glicémica del almidón y su estado microestructural. Rev. chil. nutr. [online]. 2008, vol.35, n.2 [citado 2011-10-11], pp. 84-92 . Disponible en: <http://www.scielo.cl/scielo.php?script=sci_arttext\&pid=S071775182008000200001\&lng=es\&nrm=iso>. ISSN 0717-7518.

Pérez, S. E. Efecto del consumo de una formulación que incluye almidón resistente en la glucemia postprandial de pacientes con diabetes mellitus tipo 2. Tesis de Maestría en Ciencias Alimentarias. Universidad Juárez Autónoma de Tabasco. Tabasco, México (2006).

Reyes, A. C. Caracterización fisicoquímica del almidón extraído de tres clones de plátano. Tesis de Licenciatura en Ingeniería en agroalimentos. Universidad Juárez Autónoma de Tabasco. Tabasco, México. (2000).

Rivas-González, M. Caracterización morfológica, molecular y fisicoquímica del almidón de plátano oxidado y lintnerizado. Agrociencia, 42 (5), 487-497 (2008).

Rusell, P. L., Berry, C. S., and Greenwell, P. Characterization of Resistant Starch from Wheat and Maize. Journal of Cereal Science 9: 9 -14 (1988).

Torres, G. A. y Herrera, L. Intolerancia a la glucosa, nueva entidad patológica. pp 8-14 Bayer de México, S. A. de C. V. (2005).

Tovar, J. Generalidades del Almidón. Memorias del Curso: actualización en Química y Nutrición del Almidón. 7-12. Yautepec, Morelos. México (2001).

Trinder, P. Determination of glucosa in blood using glucode oxidase with an alternative oxygen acceptor. Ann Clin Biochem 6:24. (1969).

Villada, H. S; Acosta, H. A. y Velasco, R. J. Investigación de Almidones Termoplásticos, Precursores de Productos Biodegradables. Inf. tecnol. [online]. 2008, vol.19, n.2 [citado 2011-1013], pp. 3-14. Disponible en: <http://www.scielo.cl/scielo.php?script=sci_arttext\&pid=S071807642008000200002\&Ing=es\&nrm=iso>. ISSN 0718-0764. doi: 10.4067/S0718-

Wartofsky, L. MKSAP 13. Medical Knowledge Self-Assessment Program. 42-46, Ed. American College of Physicians (2004).

Wicks, R., Trevena, L., Quine, S. Experiences of food insecurity among urban soup kitchen consumers: Insights for improving nutrition and well-being. Journal of the American Dietetic Association 106(6), 21-924 (2006).

Wolever, M. S., y Brand M., Sugar and glucose control. Am. J. Clin. Nutr. 62:212-227 (1995).

Yue, P. y Waring, S., Resistant starch in food applications. Cereal Food World. 43: 690-695 (1998). 
